\section{The extrinsic factors affecting patient access, referral and treatment of lung cancer in selected oncology public health facilities in KwaZulu-Natal}

\author{
Zondo Sinenhlanhla N.,' ${ }^{1}$ Ginindza \\ Themba G., ${ }^{2}$ Hlongwana Khumbulani W. ${ }^{2}$ \\ ${ }^{1}$ School of Social Work and Social \\ Policy, Faculty of Humanities and Social \\ Sciences, University of Strathclyde, \\ Glasgow, United Kingdom; ${ }^{2}$ Discipline \\ of Public Health Medicine, School of \\ Nursing \& Public Health Medicine, \\ College of Health Sciences, University of \\ KwaZulu-Natal Durban, South Africa
}

\section{Abstract}

Patients' insights into the factors affecting access, referral and treatment of lung cancer in public healthcare facilities, are key to the fight against this disease. On the other hand, the lack of epidemiological information on the actual burden of lung cancer, makes it difficult for public healthcare facilities to be adequately equipped to deal with foreseeable challenges in the oncology units. In order to inform patientcentred interventions and lung cancer control programmes, this study explored extrinsic factors that affect lung cancer patients throughout the cancer care continuum in KwaZulu-Natal. Nineteen patients receiving care in the three public healthcare facilities providing oncology services in KwaZulu-Natal, were interviewed using an interview guide. Following data saturation, thematic analysis was used to develop the themes relating to the extrinsic factors affecting the provision of cancer care. Five themes emerged from the analysis, namely: geographical dynamics affecting patient access to a healthcare facility, lung cancer suspicion index in the diagnostic trail within the different levels of care, decision-making trail for the referral system, equipmentinduced delays and healthcare-patient communication. A number of lung cancer patients utilising oncology services in public healthcare facilities in KwaZulu-Natal, are often met with various extrinsic factors affecting their progression through different stages of cancer care continuum. Therefore, the results of the extrinsic factors explored in this paper through the lung cancer patients' lenses may be used for designing mitigation plans to reduce delays in lung cancer care in KwaZulu-Natal.

\section{Introduction}

Non-communicable diseases such as cancer is reported to be much higher in high-income countries, ${ }^{1}$ however it is among the top causes of death in South Africa. The continuous increase of cancer morbidity and mortality in many lowincome African countries may be indicative of weak cancer programmes, low resource allocation and fragmented health care systems in the continent. ${ }^{2}$ Although efforts, such as tobacco control interventions implemented in South Africa, have decreased the overall mortality rates of cancer of the lung over the years, the cancer numbers recorded continue to reel. ${ }^{3,4}$ This rise in cancer morbidity and mortality further strains the public healthcare resources and efforts, which, in addition to cancer, should be deployed to other competing diseases priorities, including the communicable diseases. ${ }^{5}$

Early disease detection and access to treatment are paramount in the fight against lung cancer and that these are largely reliant on public health resources deployed to the oncology units in South Africa's health facilities. Achieving early disease detection and access to treatment have proven difficult in a country with disparities to access to specialized care. ${ }^{2}$ After twenty-five years of democracy and concerted efforts to improve healthcare for all, access to sufficiently resourced primary healthcare remains a topic of intense debate, ${ }^{6,7}$ yet early disease detection and screening at a primary care level could aid mitigate the growing cancer mortality rates. ${ }^{2}$ However, diagnostic delay in primary care level is one of the most notable barriers to cancer care, and this may be related to the lack of resources, low suspicion index for lung cancer and delays in referral trails. ${ }^{2,8-10}$

There is a lack of empirical studies on lung cancer care in KwaZulu-Natal in particular and South Africa in general. The gap in literature makes it difficult to determine the burden of disease, which dims the light of public healthcare efforts. With only three health facilities providing specialized diagnostic and treatment services to a large pool of various cancer cases in KwaZulu-Natal province, these facilities may not be adequately prepared. ${ }^{11,12}$ The aim of this study was to explore extrinsic factors affecting patient access, referral and treatment of lung cancer in selected public health facilities' oncology units in KwaZulu-Natal, from the perspectives of lung cancer patients receiving care in these facilities.
Correspondence: Sinenhlanhla N. Zondo, School of Social Work and Social Policy, Faculty of Humanities and Social Sciences, University of Strathclyde, Glasgow, United Kingdom.

E-mail: sinenhlanhla.zondo@strath.ac.uk

Key words: Lung cancer; access; referral; treatment; KwaZulu-Natal.

Acknowledgements: We thank KwaZuluNatal Department of Health and the brave lung cancer patients for allowing us to implement the study. We also thank the BristolMyers Squib Foundation (BMSF) "Secure the Future" for funding this project. Lastly, we are highly appreciative to the University of KwaZulu-Natal and Multinational Lung Cancer Control Programme for hosting and supporting the project.

Contributions: SZ conceptualisation, data collection and analysis and manuscript writing. $\mathrm{KH}, \mathrm{TG}$ assisted with conceptualisation, manuscript writing and revision of all drafts.

Conflict of interest: The authors declare no potential conflict of interests.

Funding: The work was supported by BristolMyers Squib foundation, Secure the Future, grant no. 10111.

Received for publication: 6 October 2021

Revision received: 6 December 2021.

Accepted for publication: 14 December 2021

This work is licensed under a Creative Commons Attribution 4.0 License (by-nc 4.0)

(C) Copyright: the Author(s), 2022

Licensee PAGEPress, Italy

Healthcare in Low-resource Settings 2022; 10:10170

doi:10.4081/hls.2022.10170

\section{Materials and Methods}

\section{Study design}

A qualitative research method was used to explore the lung cancer patients' perspectives of the extrinsic factors affecting cancer care in three hospitals offering oncology health care services in KwaZulu-Natal. A constructivist approach was used to explore the themes, as this approach gave an allowance for the participants to narrate the factors affecting their health and disease through their experiences of living with and receiving care for lung cancer. This approach has proven useful in the understanding of political, social, economic and cultural factors meshed with health and disease. ${ }^{13}$ An adapted ecological model was used to explore the different levels of influence relating to access, referral and treat- 
ment within and between the public healthcare facilities (Figure 1). The extrinsic factors explored were at the macro level of the framework, which looks at the friends and family support and public healthcare factors influencing their care. ${ }^{14}$

The study was conducted from the three healthcare facilities offering oncology services in KwaZulu-Natal. Hospital A offers specialized services including an oncology department with radiotherapy and chemotherapy, whereas Hospital B accepts patients strictly on referral basis and attends to approximately 140 new patients and 500 follow-up patients each month. On the other hand, Hospital $\mathrm{C}$ receives nationwide referrals and has highly specialized services, including pathology laboratory, radiation oncology and chemotherapy. These three hospitals were selected for the study on the basis of being the only public healthcare facilities in KwaZulu-Natal offering oncological services. Furthermore, the hospitals vary in services according to the South African Department of Health's regulation of categories of hospitals. ${ }^{15}$

The study population consisted of inpatients and outpatients diagnosed with and/or treated for lung cancer from the three facilities. The sampling strategy followed was purposive heterogeneous technique, aimed at recruiting participant from different age groups, genders, races, socio-economic and geographical backgrounds, as well as disease progression, in order to obtain various perspectives on the research question. Recruitment for data collection was done from October 2018 to January 2019, where potential participants were identified prior and after completing their consultations with medical specialists. Some participants opted to have a spouse or relative present with them throughout the interview, which assisted in the cases where participants had forgotten dates or important details pertinent to the study questions. However, questions seeking participant's perspectives were only answered by the patient. Given the nature of the study, the presence of a spouse or relative was not considered to have any serious negative effect on the quality of data generated.

All interviews were conducted in isiZulu and English, using an interview guide with open ended questions. All interviews conducted in isiZulu were translated by a professional transcriber into English after verbatim transcription of audio-files by the transcriber had been concluded.

This study was approved by the UKZN Biomedical Research Ethics Committee (BE 534/18) and KwaZulu-Natal Department of Health (KZ_201801_013) on 14 September 2018 and 03 October 2018, respectively. Potential participants were informed about the purpose of the study prior to signing informed consent forms and conducting an in-depth interview.

The lead researcher, who is a qualitative data analysis expert with 3 years of experience, coded and analysed the data using NVIVO software. Co-authors reviewed the analysis and raised queries, where necessary, which helped the lead researcher con- sistently reflect on all the factors that affected her analysis. Data analysis was performed using thematic analysis following the process described by Braun and Clarke. ${ }^{16}$ During the thematic analysis process the transcripts were read in detail, initial codes were generated then collated into potential themes. Once initial themes were generated, they were reviewed and refined to ensure that they addressed the study objectives. Lastly themes were developed and discussed by all the authors to reach consensus.

\section{Results}

The ages for the 19 participants ranged from 43 to 80 years, reflecting a fairly diverse mix of genders, ethnicity and residential areas (Table 1).

\section{Identified themes}

Five themes emerged from the analysis. These themes were: the dynamics affecting patient access to healthcare facility, lung cancer suspicion index in the diagnostic trail within the different levels of care, healthcare provider-patient communication, decision-making trail for the referral system and equipment-related delays. Themes were further located within the continuum of cancer care, including access to oncology care, referral system for lung cancer patients and treatment services for lung cancer.

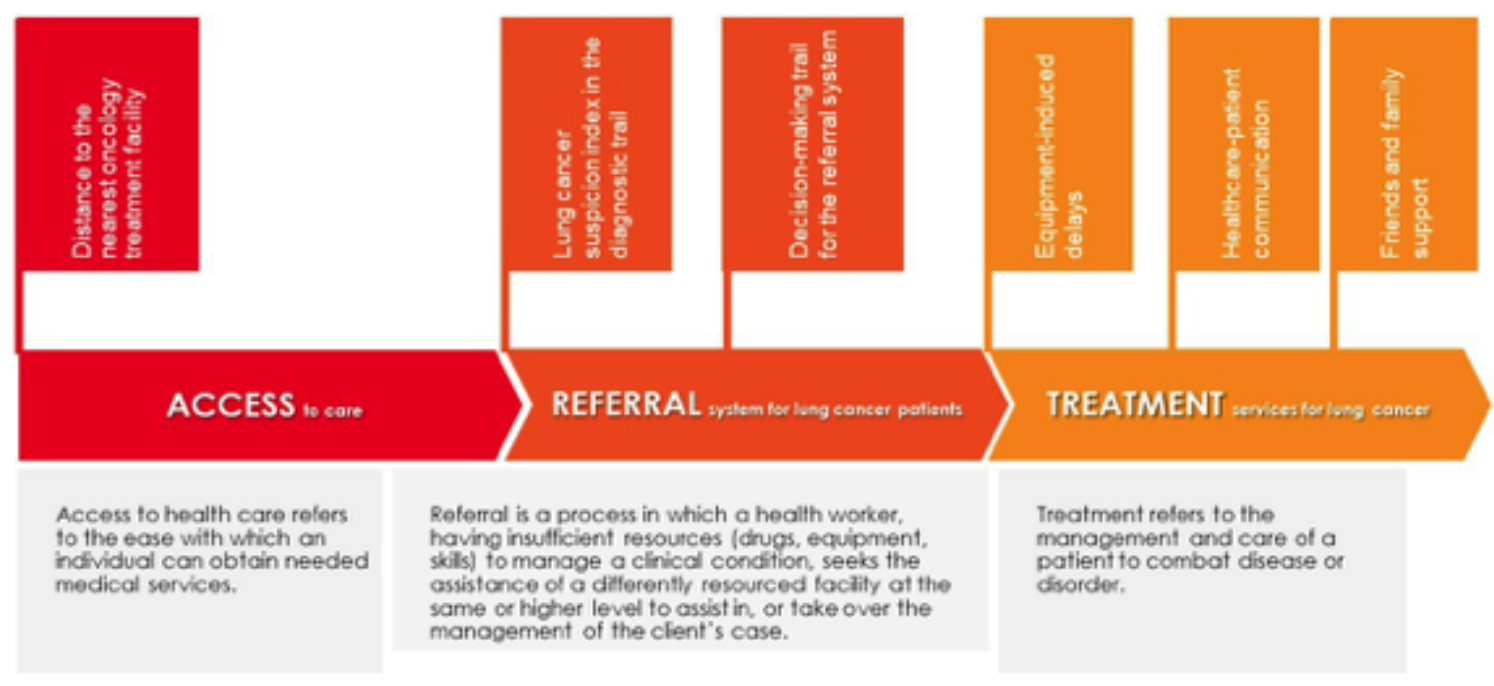

Figure 1. Multilevel influences on access, referral and treatment of lung cancer in oncology public health facilities. Extracted and adapted from Taplin et al., 2012. ${ }^{14}$ 


\section{Access to oncology care}

\section{Dynamics affecting patient access to healthcare facility}

Results of this study revealed that access to healthcare facilities is still a challenge for many patients living with cancer. There was a convergence of views in lung cancer patients' identification of the distance and means to the nearest oncology treatment facility as the main barriers to receiving appropriate care. In addition to the lack of appropriate care at the primary care facility, poor means to health facilities further affect the health of the patients.

"When I leave home, I use the cars from the community, then I go to St. William's [hospital]. I then sleep over at St. Williams, and then from there I travel by an ambulance that is coming here [Greenwood hospital] ... Today we will leave in the afternoon, then I will sleep over at the hospital again and then I will take a taxi that is going back home" 79-year-old female

"I travel by foot, hmm one and a half, one and a half... maybe two hours...You see, just like now it's very difficult. I must get a car that will take me, and then sometimes I will send someone to collect the pills for me. I go sometimes when I have to go to check my weight or for BP check-up, I try to get transport to go there but when I get back I am going to be sick the whole week. It's really difficult to walk long distances" 55year-old male

"Then I was complaining to the Doctor, I was coughing blood, he didn't want to attend to me. He took an X-ray and he didn't want me to see what's going on. So, they sent me back to Deansdale [hospital]. When I went to Deansdale they took the blood tests and they found that there's a cancer right there in my lungs. So, when I came here [oncology clinic] it was too late man. It couldn't be stopped, it already went to my lungs. " 55-year-old male

\section{Referral system for lung cancer patients}

Lung cancer suspicion index in the diagnostic trail within the different levels of care

Given the overlapping symptoms between $\mathrm{TB}$ and lung cancer, healthcare workers were prone to suspecting TB, negating the possibility of lung cancer, which is sometimes viewed by participants as guesswork. Participants perceived the lung cancer suspicion index by healthcare providers to be low. The lack of extensive diagnostic tools in many facilities exacer- bates the problem.

"They [healthcare providers] were saying TB, maybe it's a growth, maybe it's cancer, maybe.... They were guessing" 55-yearold male

"No symptoms whatsoever, just the sore throat uhmm and then I started this incredible pain in my chest here, just shooting. But it wouldn't last long. It would just last for a while and then go away. But when it [the pain] was there, it was so intense that it really, you know, it forced me to go to the Doctor. He diagnosed me with Bornholm disease. But uhm, he said that he wanted to take X-rays and bloods, just in case." 52year-old female

"I was coughing and then I went for an $X$-ray in the rural area near home, here in eThekwini. When I did the X-ray, they said it's TB. I took the TB treatment then while I was still taking it they said I must come back after a month and then I went back. When I went back for an X-ray they said no... they gave me a letter and told me to go to.... they said I must go to Marshall[hospital]. So, at Marshall I did an X-ray there and they said I have cancer" 80-year-old female

Decision-making trail for the referral system

Participants expressed that obtaining referral note to the necessary facility was a factor and when a referral note was eventually received, the wait time before seeing a specialist lasted for a few months. This delay may have been as a result of the large number of new and returning cancer patients in these three facilities.

"What happened is that I was made to wait, and I noticed that it [growth] was continuing. It's the Doctor that speed up the process because I didn't get much help from the clinics." 71-year-old female

"I was coughing on and off in 2016. In three months', time I was referred from Ghandi to here [hospital with oncology services]." 48-year-old male

"I once went to check for cancer at the clinic and when I got back from the checkup, they [healthcare providers] said that my cancer was not yet critical, so they were prioritising those who are critical to receive treatment. So, each time I would go they would tell me it wasn't bad until they finally told me when they did." 80-year-old female

\section{Treatment services for lung cancer}

\section{Equipment-induced delays}

Lack of functioning diagnostic and treatment equipment was flagged as an
Table 1. The demographic characteristics of the study participants $(n=19)$.

\begin{tabular}{lc} 
Characteristics & n (\%) \\
AGE & \\
Mean & 64.37 \\
Range (SD) & 11.68 \\
GENDER & \\
Female & $8(42.1)$ \\
Male & $11(57.89)$ \\
\hline ETHNICITY & \\
Black & $10(52.63)$ \\
Indian & $6(31.58)$ \\
Coloured & $1(5.26)$ \\
White & $2(10.53)$ \\
RESIDENTIAL AREA & \\
Rural & $7(36.84)$ \\
Township & $9(47.37)$ \\
Suburban & $3(15.79)$ \\
\hline HOSPITAL & \\
A & $5(26.32)$ \\
B & $7(36.84)$ \\
C & $7(36.84)$ \\
\hline
\end{tabular}

issue that immensely contributed to treatment delays and accumulation of waiting list. This ranged from x-ray machinery to radiotherapy machinery.

"These machines are always broken and whatever. And I needed, because of the aggressive nature of my cancer I needed to get the results sooner rather than later. And I managed, through the grace of God and through a previous oncologist that was here to get in, because there is a very long waiting list to get in here" 52-year-old female

"I started coming here [Greenwood hospital] last year because they said they don't have machines at Woodlands [hospital], so I would get the machines here." 74year-old male

"I started going for check-ups in 2005 until 2010, then they only discovered in 2011 that I have cancer. They transferred me to King George hospital and I stayed about two months or about one month and three weeks. They said they suspect that I have lung cancer but now their machines are not sufficient, so they sent me to King's. I stayed for two months. They would check me while I was admitted, then on the third month they said that they found that I have lung cancer, my lung was now damaged so they asked for my permission to remove the lung..." 55-year-old male

\section{Healthcare provider-patient commu- nication}

Communication between patients and their Healthcare Providers (HCPs) may be a challenge on the extent to which the patient 
understands how the disease manifests and adherence to treatment methods thereof. Communication barriers may result from language, different cultural backgrounds and at times the patient's lack of knowledge of technical concepts. Patients were in agreement in that, even after being diagnosed and undergone treatments, they still did not have detailed knowledge about their illness. This may be indicative of poor healthcare provider-patient communication regarding the patient health issues.

"This year once again now, I was telling this Doctor and he is just ignoring me. He referred me back to Deansdale [hospital]. And in Deansdale the results said I must come back here because it's cancer, they can't do nothing. They don't work with cancer." 55-year-old male

"Yes. They said they found that it's cancer, but they didn't explain what type of cancer it was" 43-year-old male

"Look, let's put it this way, you are pretty much in the dark when it comes to this. They say you have got to go for chemo, but nobody can tell you the side effects because everybody is affected differently so, you are pretty much in the dark. It's just you go for chemo and take it from there" 73-year-old female

One participant stated that he was booked for a biopsy but was suddenly told he was getting his whole left lung removed just before he was given the consent form to sign.

"...and then they said to me, no they will just take a small piece of my lung out. So, you know you must sign a form of consent before you go into the theatre. The Doctor came to me and he said "Mr *Armstrong [not his real name] listen here, this cancer has spread right through your lung, so I'm gonna take the whole lung out". He said 'Mr Armstrong you don't like to sign this here but, in a few months, you come back to me and this lung, this cancer will spread right through. That's why I'm telling you this now. That's why I'm giving you this consent to sign'. So, I thought to myself, bring the form, let me sign." 52year-old male

The above illustration presents a summary of the extrinsic factors, categorised into five thematic areas (Figure 2), affecting access, referral and treatment identified by lung cancer patients in this study.

\section{Discussion}

Access to healthcare facilities is the most crucial stage in the patient's care continuum, because it directs the route the cancer care continuum ought to take. ${ }^{17}$ Substantial geographic differences and their effect on access to healthcare facilities are especially evident in the results of this study. The proximity to regional hospitals appeared to particularly help patients in receiving a relatively quicker diagnosis, as opposed to their counterparts who had to travel long distances to the nearest healthcare facility. In a country, such as South Africa, where socioeconomic disparities are often swayed by one's ethnicity, healthcare facilities are still placed in areas predominately populated with White, Coloured and Indian people, ${ }^{6,7,18}$ thereby inadvertently prejudicing the majority of Black South Africans, who were placed by the previous government regime in peripheral areas. ${ }^{6} \mathrm{An}$ Annual General Household Survey conducted in South Africa found that vulnerable sub-groups, such as rural and/or Black South Africans had restricted access to healthcare in South Africa, despite the implementation of various health reforms since the abolishment of apartheid laws. ${ }^{6}$ While rural areas may have well-functioning clinics, these establishments are not

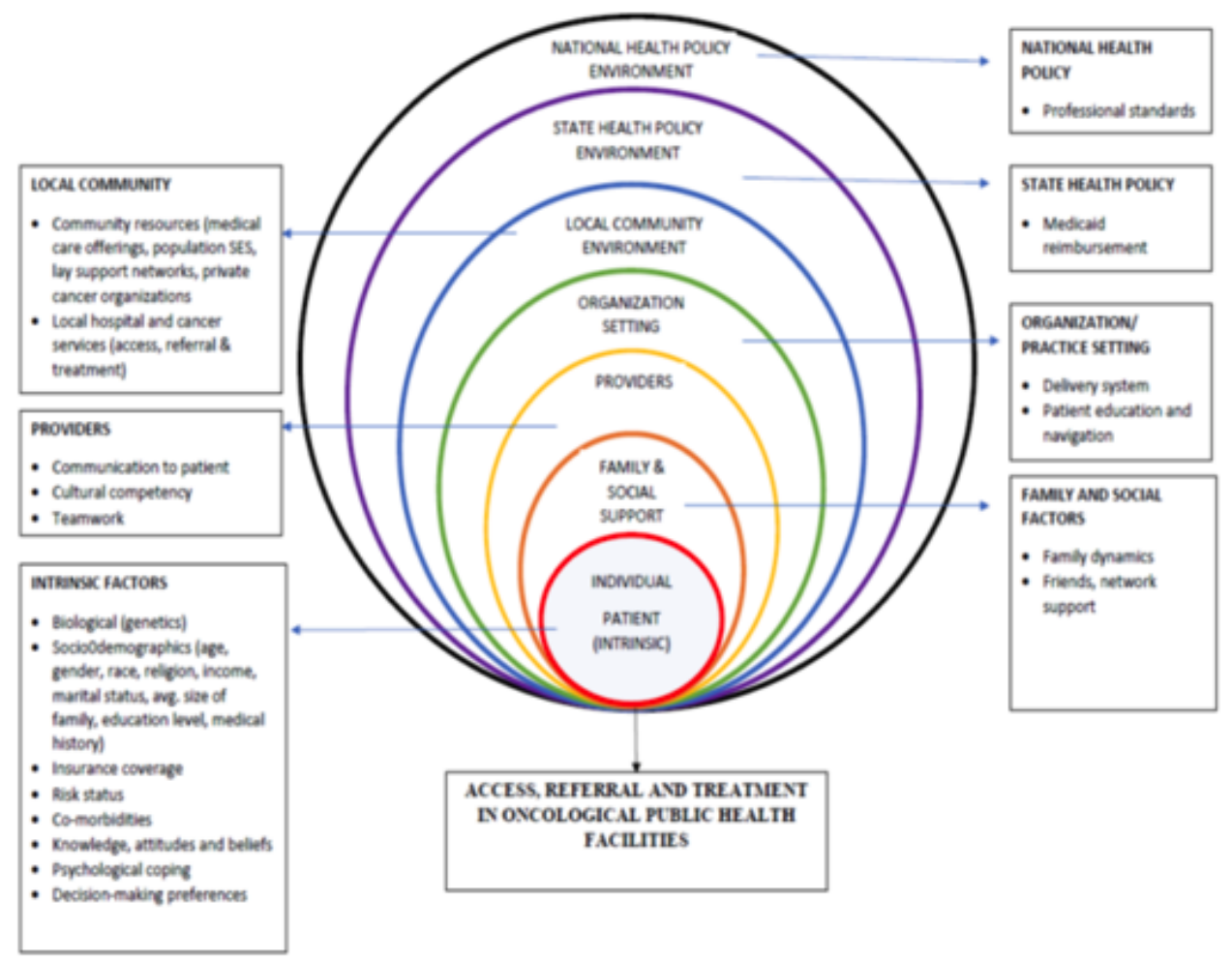

Figure 2. Extrinsic factors affecting lung cancer care in KwaZulu-Natal. 
adequately equipped to work with complex illnesses, using intricate diagnostic resources, such as that of the lung cancer. ${ }^{17}$

Access to effective cancer care in LMICs, such as South Africa, is a complex issue compounded by various factors, often resulting in the phenomena of the inversecare law. ${ }^{7}$ The 'inverse-care law' is a term defined as the inverse relationship between the availability of effective medical care and the population needs. ${ }^{7}$ In a country with extreme poverty margins, such as those seen in rural and township areas, health is affected by access to basic requirements for life. These basic requirements include adequate nutrition, access to vaccinations, availability of jobs and reasonable housing conditions. ${ }^{19}$ Similarly, rural patients in this study stated that they were able to access local clinics for other general illnesses, but they were unable to access proper diagnostic services for possible cancer screening until referral to a regional hospital, which was further away from their home. In addition to the unavailability of adequate care in their local clinics, transport expenses made access to healthcare largely reliant on one's geographical residential area. However, it is crucial to note that some patients from rural areas were transferred quickly to the nearest treating hospitals, but had to travel through hospital patient transportation, which operated on routine basis to accommodate other patients. In one instance, a patient recounted that she travelled from her home to the nearest regional hospital, was admitted for the night and departed the next morning for her to make her appointment at the treating hospital. This process was repeated for initial consultation, chemotherapy and or regular check-ups. For patients with an illness with low survival rates, swift interventions are paramount and travelling long distances is not suitable. ${ }^{7}$

Congruent with the findings of this study, literature has shown that the incorrect diagnosis is one of the leading causes of the referral delays to cancer care in Africa. ${ }^{10,20}$ These results are further supported by Masamba's study, which revealed that misdiagnosis of lung cancer as tuberculosis remains a major concern for Low-MiddleIncome Countries (LMICs). ${ }^{20}$ This is attributable to the fact that lung cancer is not a common form of cancer seen in the primary healthcare facilities and often has no clear signs and symptoms recognisable by the patient and at times, not even by the healthcare workers. ${ }^{10}$ Lung cancer also shares a few symptoms common with Tuberculosis (TB), such as chest pains and coughing blood sputum. ${ }^{20}$ With the high incidence rate of TB in South Africa, it is relatively easy to misclassify malignancies, such as lung cancer and lymphoma for TB, especially when appropriate diagnostic measures are not utilised. ${ }^{10}$ This delay in correct diagnosis is detrimental for the patient's overall health outcomes, as it exposes the patient to unnecessary TB drug resistance.

Incorrect diagnosis may also be as a result of the lack of cancer literacy amongst primary care healthcare providers or lack of suitable diagnostic tools. ${ }^{10,21}$ Consequently, healthcare providers could unknowingly misinterpret lung cancer symptoms and commence with inappropriate treatment without a biopsy, which would have provided more accurate results. ${ }^{20}$ Patients stated that their initial contact with their Primary Care Provider (PCP) did not provide them with a correct diagnosis, thereby resulting in further delay in implementing referral and treatment protocols. A systematic review on the role of primary care in detection and follow-up of cancer highlighted the importance of reducing diagnostic delays amongst higher index suspicion cases seen by primary care providers. ${ }^{22}$ The idea of improving diagnostic tools and encouraging a fast-track referral system might be a possible answer to a growing public health concern. However, this initiative would require additional training and education for the PCPs and constant prompts and reminders in the facilities to encourage screening for various cancers when applicable. ${ }^{22}$ Although this may not completely combat the issue of misdiagnosis in primary care facilities, it might reduce the burden of screening done in regional and tertiary hospitals. Furthermore, improving diagnostic tools is likely to provide a fast-track for more symptomatic cases, which allows for an overall more resourceful referral system.

While receiving a referral did not seem to be the main obstacle for most participants, the duration between the correct diagnosis and a consultation in the oncology facility was prolonged. Some patients stated that they had to speak to specific specialists in the oncology department for quicker referral and biopsy results. This delay in referral consultations may have profound implications on the treatment. Similarly, a scoping review of health systems delays in lung cancer stated that delays in obtaining access to definitive diagnostic procedures and results is one of the main factors contributing to delays in lung cancer care. ${ }^{23}$ This was seen in a case of a lung cancer patient diagnosed in May 2017 covered by the South African newspaper 'Business Live' in 2018. ${ }^{12}$ The patient was given a follow-up date that required him to wait for 15 months for a scan in a local hospital, but succumbed to his illness after 12 months. ${ }^{12}$
This unfortunate case may characterise the many deaths that are due to delays in health systems in South Africa.

Equipment-induced delays are at the crux of cancer care barriers in LMICs in Africa $^{24}$ and South Africa is no exception. ${ }^{12}$ One of the recurring themes in this study pertained to the delays relating to lack or breakage of equipment for diagnostic and treatment purposes in at least one of the three participating facilities. South African Regional hospitals are expected, but not obligated to provide oncology services as a part of the specialisation departments, leaving KwaZulu-Natal with just two tertiary hospitals, which have mandatory oncology services. $^{15}$ This imbalance between resources and cancer patients poses a threat to the number of individuals requiring radiotherapy and chemotherapy daily in these hospitals. ${ }^{12}$ Procurement of the stateof-the-art equipment is often not feasible in African countries, where budget allocations are skewed towards communicable disease control and corruption exacerbates the scarcity of resources spent on each patient. ${ }^{25}$ Participants shared experiences of having to be referred to various regional hospitals for confirmation of diagnosis and treatment, which prolonged the delay, ultimately leading to advancement of disease, thereby limiting the treatment options available. ${ }^{26}$ The lack of radiotherapy equipment is a factor to be considered in the battle against cancer in Africa, because, as many as $50 \%$ of cancer cases would benefit from radiotherapy treatment. ${ }^{12}$

The longstanding good physicianpatient communication model has proven to yield better health outcomes in different settings. ${ }^{24,27}$ However, poor physician-patient communication was one of the factors that affected the lung cancer treatment adherence in South Africa. There is a shift from passive recipients of advice to more wellinformed consumers who actively engage with decisions about their health. ${ }^{27}$ This is an idealistic picture of HCP-patient communication; however, this is hardly achieved in many LMIC settings. Patients expressed that they were often in the dark about the stage of their cancer, cancer type and treatments being provided. The example of a patient admitted for a lung biopsy and being informed of a full lung removal just before the surgery, does not exhibit good HCP-patient communication, as the options available to the patient need to be fully explained. A systematic review exploring patients' experiences in communicating with Primary Care Physicians (PCPs) identified ineffective communication between healthcare providers and patients as the cause of stress, helplessness and dominance 
of biomedical interventions, which negatively affected adherence to treatment. ${ }^{28,29}$ The concept of a patient navigator, should be considered. Patient navigators are able to simplify specialist information when communicating with the patient. This is also a person who may follow-on the progress of the patient.

\section{Conclusions}

Extrinsic factors affecting lung cancer patient access, referral and treatment in KwaZulu-Natal was due to difficulty in accessing adequate healthcare facilities, low lung cancer suspicion index in the diagnostic trail within the different levels of care, weak healthcare provider-patient communication, referral delays and equipmentinduced delay. The results of this study are consistent with the findings of a study conducted in the Limpopo province, which highlighted communication and equipmentinduced problems with follow-up care, as important factors influencing cancer treatment in the province..$^{30}$ These findings may be useful in highlighting pertinent oncology care issues in South Africa and aid in the improvement of facilities to adequately deal with lung cancer cases in KwaZulu-Natal. Additionally, this study highlighted the importance of timely diagnosis, which is eminent in successful lung cancer treatment.

\section{References}

1. Nojilana B, Bradshaw D, Pillay-van Wyk V, et al. Persistent burden from non-communicable diseases in South Africa needs strong action. South Afr Med J 2016;106:436-7.

2. Cazap E, Magrath I, Kingham TP, Elzawawy A. Structural barriers to diagnosis and treatment of cancer in low- and middle-income countries: The urgent need for scaling up. J Clin Oncol 2016;34:14-9.

3. WHO. New Global Cancer data: GLOBOCAN 2018. WHO, 2018. Available from: https://www.uicc.org/new-global-cancer-data-globocan-2018\#

4. Winkler V, Mangolo NJ, Becher H. Lung cancer in South Africa: a forecast to 2025 based on smoking prevalence data. BMJ Open 2015;5:e06693.
5. Mayosi BM, Flisher AJ, Lalloo UG, Sitas F, Tollman SM, Bradshaw D. The burden of non-communicable diseases in South Africa. Lancet 2009;374:93447.

6. Burger R, Christian C. Access to health care in post-apartheid South Africa: Availability, affordability, acceptability. Health Econ Policy Law 2018:1-13.

7. Harris B, Goudge J, Ataguba JE, McIntyre D, Nxumalo N, Jikwana S, et al. Inequities in access to health care in South Africa. J Public Health Policy 2011;32:S102-23.

8. Moodley J, Walter FM, Scott SE, Mwaka AM. Towards timely diagnosis of symptomatic breast and cervical cancer in South Africa. S Afr Med J 2018;108:803-804.

9. Espina C, McKenzie F, dos-SantosSilva I. Delayed presentation and diagnosis of breast cancer in African women: a systematic review. Ann Epidemiol 2017;27:659-71.e7.

10. Hannan A. Misdiagnosis of cancer as tuberculosis in low- to middle-income countries: A tip of the iceberg! J Glob Oncol 2016;2:244-5.

11. Morhason-Bello IO, Odedina F, Rebbeck TR, et al. Challenges and opportunities in cancer control in Africa: a perspective from the African Organisation for Research and Training in Cancer. Lancet Oncol 2013;14:14251.

12. Mnyanda L. Care for cancer patients lags behind in SA's public health facilities. Business LIVE. 27 June 2018.

13. Baum F. Researching public health: Behind the qualitative-quantitative methodological debate. Social Sci Med 1995;40:459-68.

14. Taplin SH, Anhang Price R, Edwards $\mathrm{HM}$, et al. Introduction: Understanding and influencing multilevel factors across the cancer care continuum. J National Cancer Institute Monographs 2012;2012:2-10.

15. Health Do. Government notices: regulations relating to categories of hospitals. In: Health, editor. 2012.

16. Braun V, Clarke V. Using thematic analysis in psychology. Qual Res Psychol 2006;3:77-101.

17. Venter M, Venter C, Botha K. Cancer treatment in South Africa: A narrative literature review. J Psychol Afr 2012;22:459-66.

18. Coovadia H, Jewkes R, Barron P, et al.
The health and health system of South Africa: historical roots of current public health challenges. Lancet 2009;374:817-34.

19. Mayosi BM, Benatar SR. Health and Health Care in South Africa - 20 Years after Mandela. New Engl J Med 2014;371:1344-53.

20. Masamba LPL, Jere Y, Brown ERS, Gorman DR. Tuberculosis diagnosis delaying treatment of cancer: Experience from a new oncology unit in Blantyre, Malawi. J Global Oncol 2016;2:26-9.

21. Mansell G, Shapley M, Jordan JL, Jordan K. Interventions to reduce primary care delay in cancer referral: a systematic review. $\mathrm{Br} \mathrm{J}$ Gen Pract 2011;61:e821-e35.

22. Emery JD, Shaw K, Williams B, et al. The role of primary care in early detection and follow-up of cancer. Nature Rev Clinical Oncol 2013;11:38.

23. Malalasekera A, Nahm S, Blinman PL, et al. How long is too long? A scoping review of health system delays in lung cancer. Eur Resp Rev 2018;27:180045.

24. Nonzee NJ, Ragas DM, Ha Luu T, et al. Delays in Cancer Care Among LowIncome Minorities Despite Access. J Women's Health 2015;24:506-14.

25. Mostert S, Njuguna F, Olbara G, et al. Corruption in health-care systems and its effect on cancer care in Africa. Lancet Oncol 2015;16:e394-e404.

26. O'Rourke N, Edwards R. Lung cancer treatment waiting times and tumour growth. Clinical Oncology 2000;12:141-4.

27. Diefenbach M, Turner G, Carpenter $\mathrm{KM}$, et al. Cancer and patient-physician communication. J Health Comm 2009;14:57-65.

28. Rocque R, Leanza Y. A systematic review of patients' experiences in communicating with primary care physicians: Intercultural encounters and a balance between vulnerability and integrity. PloS One 2015;10:e139577e.

29. Frankel RM. Relationship-centered care and the patient-physician relationship. J General Internal Med 2004;19:1163-5.

30. Ramathuba D, Jacqueline R, Ndou N. Patients' perspective of cancer treatment and care in Vhembe District of Limpopo Province. J Nurs Care 2014;3:2167-1168.1000186. 